\title{
Two-dimensional hole precession in an all-semiconductor spin field effect transistor
}

\author{
Marco G. Pala \\ Institut für Theoretische Festkörperphysik, \\ Universität Karlsruhe, D-76128 Karlsruhe, Germany and \\ Dipartimento di Ingegneria dell'Informazione, \\ via Diotisalvi 2, I-56126 Pisa, Italy \\ Michele Governale \\ Institut für Theoretische Festkörperphysik, \\ Universität Karlsruhe, D-76128 Karlsruhe, Germany and \\ NEST-INFM \& Scuola Normale Superiore, \\ piazza dei Cavalieri 7, I-56100 Pisa, Italy \\ Jürgen König \\ Institut für Theoretische Festkörperphysik, \\ Universität Karlsruhe, D-76128 Karlsruhe, Germany and \\ Institut für Theoretische Physik III, \\ Ruhr-Universität Bochum, D-44780 Bochum, Germany \\ Ulrich Zülicke \\ Institut für Theoretische Festkörperphysik, \\ Universität Karlsruhe, D-76128 Karlsruhe, Germany and \\ Institute of Fundamental Sciences, Massey University, \\ Private Bag 11 222, Palmerston North, New Zealand*
}

Giuseppe Iannaccone

Dipartimento di Ingegneria dell'Informazione, via Diotisalvi 2, I-56126 Pisa, Italy

(Dated: February 2, 2008) 


\begin{abstract}
We present a theoretical study of a spin field-effect transistor realized in a quantum well formed in a p-doped ferromagnetic-semiconductor- nonmagnetic-semiconductor-ferromagneticsemiconductor hybrid structure. Based on an envelope-function approach for the hole bands in the various regions of the transistor, we derive the complete theory of coherent transport through the device, which includes both heavy- and light-hole subbands, proper modeling of the mode matching at interfaces, integration over injection angles, Rashba spin precession, interference effects due to multiple reflections, and gate-voltage dependences. Numerical results for the device current as a function of externally tunable parameters are in excellent agreement with approximate analytical formulae.
\end{abstract}

PACS numbers: 85.75.Hh, 72.25.-b, 73.23.Ad 


\section{INTRODUCTION}

Spintronics has attracted great interest in the scientific community, ${ }^{1.2}$ advocating the use of the spin degree of freedom in electronic devices. Combining this idea with mesoscopic transport has stimulated investigations of coherent spin-dependent phenomena. Many proposed device setups exploit the effect of spin-orbit coupling on the carrier motion. 3.4.5.6.7.8.9 The most popular proposal for a coherent spintronic device is the spin field-effect transistor (spin FET) proposed by Datta and Das. ${ }^{10,11}$ It consists of a two-dimensional (2D) electron gas confined in a semiconductor heterostructure that is attached to two ferromagnetic contacts acting as source and drain. Majority-spin electrons injected from the source experience a spin precession due to the Rashba effect ${ }^{12.13 .14}$ if the magnetization direction in the source contact is parallel to the direction of current flow or perpendicular to the plane of the 2D electron gas. Tunability of the spin-orbit coupling strength by gate voltages enables external control of this spin precession and, hence, manipulation of the current transmitted at the second ferromagnetic contact. Besides gate-voltage control of the Rashba spin-orbit coupling experienced by 2D electrons, which has been successfully demonstrated

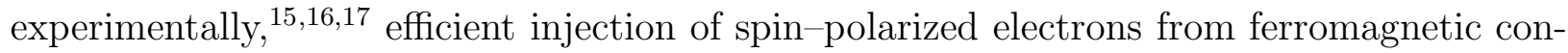
tacts into the nonmagnetic part of the spin FET is a key ingredient for device operation. The obvious challenges involved in the fabrication of hybrid systems consisting of metallic and semiconducting parts, as well as a physical limitation ${ }^{18}$ to the amount of spin injection that can be achieved in the absence of tunnel barriers at the interfaces, have so far prevented the realization of any spin FET device. A possible solution to circumvent these difficulties may be provided by the use of diluted magnetic semiconductors ${ }^{19}$ as source and drain. This motivates our present study where we investigate transport through $2 \mathrm{D}$ hybrid structures with ferromagnetic contacts realized in semiconductor heterostructures. An important aspect of our work deals with the fact that ferromagnetic (III,Mn)V compounds are intrinsically p-doped, implying that currents are carried by holes rather than electrons. The spin properties of carrier states in the intrinsically p-like valence bands of III-V semiconductors are very different from that in the s-like conduction band. To begin with, several valence bands with different effective masses exist. More importantly, however, spin-orbit coupling in the valence bands has a more complicated structure than that of conduction-band electrons. ${ }^{20,21}$

The aim of this article is a detailed study of an all-semiconductor spin field-effect tran- 


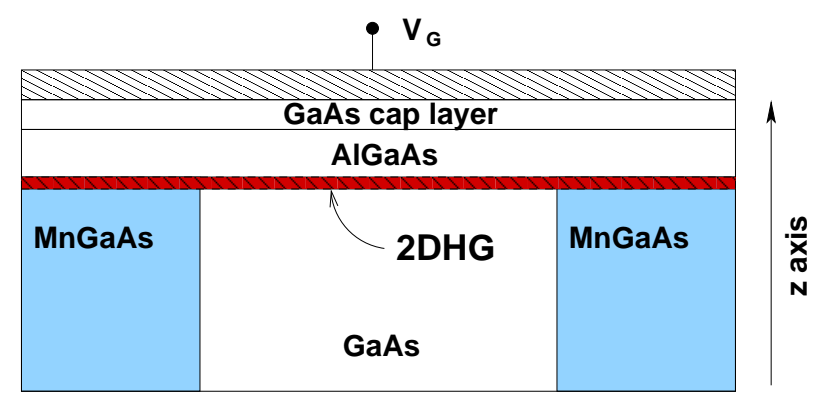

FIG. 1: Schematic illustration of the proposed device. The two-dimensional hole gas (2DHG) in the GaAs part is attached to spin-polarized source and drain contacts, formed by 2DHGs in the MnGaAs parts. The gate electrode on the top controls both the carrier concentration and the Rashba spin-orbit coupling strength.

sistor in which the conducting channel is provided by a $2 \mathrm{D}$ hole gas (2DHG). The device structure we propose is depicted in Fig. 1. A MnGaAs/GaAs/MnGaAs heterostructure is overgrown $^{22.23}$ in the $z$ direction with AlGaAs such that a 2DHG forms at the interface. In fact, Mn doping is only required within the quantum well formed at the interface to the AlGaAs layer, but the Mn ions outside the well do not disturb. In the proposed setup, source and drain are defined by 2D quantum wells accommodating spin polarized holes. The carriers in the entire 2DHG are subject to the Rashba effect which leads to spin precession. The strength of the Rashba spin-orbit coupling can be tuned by a gate voltage applied to the top of the sample. ${ }^{24}$

The key ingredient for the functionality of the device is the tunable Rashba spin precession in the 2DHG. In Ref. 25, we studied this part within a simplified model allowing for an analytic treatment, which enabled us to discuss features that are universal for both electron and hole transport. In the present paper, we aim at a more complete numerical treatment of transport through the entire device. This includes describing the semiconductor valence bands by a $4 \times 4$ Kohn-Luttiger Hamiltonian instead of restricting ourselves to heavy-hole bands only. We take into account the matching properties of the modes at the interfaces to the source and drain contacts, which automatically includes interference effects due to multiple reflection. As in Ref. 25, we allow for all possible injection angles instead of restricting to a quasi-one-dimensional setup. In our analysis we study the purely ballistic regime. Scattering due to impurities or to the lateral finite size of the device due to the functional form of the spin-orbit coupling Hamiltonian for holes affects the total angular 
momentum $\mathbf{j}$ and not only the spin. This leads to extra damping of spin-precession and should be minimized to improve device functionality. Finally, for addressing the response of transport to the gate voltage, we employ a capacitive model that takes the variation of both the carrier density and the Rashba coupling strength into account.

The article is organized as follows. We introduce, in Sec. III, the Kohn-Luttinger Hamiltonian for the ferromagnetic and nonmagnetic parts of the semiconductor quantum well. In the following Section III, we describe the mode-matching technique used to calculate transmission coefficients for transport through the structure. Our results from numerical simulations of transport are presented in Section [V] After discussing the case of only one interface between a ferromagnetic and a nonmagnetic 2DHG, we turn our attention to the full transistor geometry with two ferromagnetic contacts. In the latter case, interference effects appear due to multiple reflections at the interfaces. In Section $\nabla$, we describe an analytical model that approximates the numerical simulations very well and helps us to understand how the precession length $L_{\mathrm{so}}$ depends on the Fermi energy $E_{\mathrm{F}}$ in the 2DHG. In Section VI, we address the response to external gate voltages. The possibility to control the spin precession by a single gate voltage $V_{\mathrm{G}}$ that simultaneously modifies Fermi energy and Rashba spin-orbit coupling is discussed in detail.

\section{ENVELOPE-FUNCTION DESCRIPTION OF 2D VALENCE-BAND STATES}

In this section, we obtain effective Hamiltonians that describe the valence bands in the different regions of the spin FET, namely, the ferromagnetic source and drain contacts doped with $\mathrm{Mn}^{2+}$ ions and the undoped nonmagnetic channel in between. In all these regions, holes are confined within a 2D quantum well. We use an envelope-function description ${ }^{26.27 .28}$ of the $2 \mathrm{D}$ system. The Hamiltonian for the nonmagnetic semiconductor $H_{\mathrm{p}}$ is the sum of a $2 \mathrm{D}$ quantum-well Hamiltonian $H_{2 \mathrm{D}}$ plus a Rashba term $H_{\mathrm{rs}}$, which arises due to the asymmetry of the confinement potential $V_{\text {con }}(z)$. On the other hand, the total Hamiltonian for the ferromagnetic contacts $H_{\mathrm{f}}$ is given by $H_{\mathrm{p}}$ plus the term $H_{\mathrm{pd}}$, which takes into account the coupling between the p-like valence holes and the half-filled d-shell $\mathrm{Mn}^{2+}$ ions with spin $S=5 / 2$.

Bulk systems host heavy- and light-hole bands with total angular momentum $j=3 / 2$. These bands are degenerate at the band edge and are well separated, due to spin-orbit 
coupling, from the split-off bands with total angular momentum $j=1 / 2$. In quantum wells, each of these bands is transformed in a sequence of quasi-2D subbands, and the degeneracy between heavy and light-hole bands is lifted. In the following, we are interested in the situation at low carrier concentrations such that only the lowest subbands, one heavy-hole band (HH1) and one light-hole band (LH1), have to be taken into account. This is the simplest realistic case that occurs when the triangular quantum well is narrow enough to sufficiently lift the energy of higher subbands. Inclusion of higher energy subbands, in particular HH2, would be straightforward, but would only lead to higher order corrections. Indeed, the low hole density typically present in experiments implies that the only occupied propagating modes are in HH1, whose shape is influenced by the band mixing ${ }^{26}$ with LH1 and in a negligible way with HH2. Coupling with HH2 would affect the shape of LH1, and therefore only evanescent modes. In the basis of total angular momentum,

$$
\begin{aligned}
& |1\rangle=\left|j=3 / 2, j_{z}=3 / 2\right\rangle \\
& |2\rangle=\left|j=3 / 2, j_{z}=-1 / 2\right\rangle \\
& |3\rangle=\left|j=3 / 2, j_{z}=1 / 2\right\rangle \\
& |4\rangle=\left|j=3 / 2, j_{z}=-3 / 2\right\rangle
\end{aligned}
$$

the Hamiltonian $H_{2 \mathrm{D}} \operatorname{reads}^{26}$

$$
H_{2 \mathrm{D}}=\left(\begin{array}{cccc}
h h & d & 0 & 0 \\
d^{*} & l h & 0 & 0 \\
0 & 0 & l h & d \\
0 & 0 & d^{*} & h h
\end{array}\right)
$$

with

$$
\begin{aligned}
& h h=E^{\mathrm{hh} 1}+\frac{\hbar^{2}}{2 m_{\mathrm{hh} \|}} k^{2} \\
& l h=E^{\mathrm{lh} 1}+\frac{\hbar^{2}}{2 m_{\mathrm{lh} \|}} k^{2} \\
& d=-\frac{\sqrt{3} \hbar^{2}}{2 m} \bar{\gamma} N k^{2} e^{-i 2 \alpha} .
\end{aligned}
$$

Here we have adopted the momentum-space representation in polar coordinates for the wave vector $\mathbf{k}_{\|}=(k \cos \alpha, k \sin \alpha)$ in the $2 \mathrm{D}$ plane. The quantities $E^{\mathrm{hh} 1}$ and $E^{\mathrm{lh} 1}$ in Eq. (3) are the subband-bottom energies deriving from the solution of the Schrödinger problem for the triangular well, and the factor $N$ takes into account the scalar product between the envelope functions $f^{\text {hh1,lh1 }}$ for the HH1 and LH1 subbands. We observe that in Eq. (2) there is no coupling between LH and HH subbands with the same sign of $j_{z}$ since the corresponding matrix element is proportional to the vanishing integral $\left\langle f^{\mathrm{hh} 1}\left|k_{z}\right| f^{\mathrm{lh} 1}\right\rangle$. 


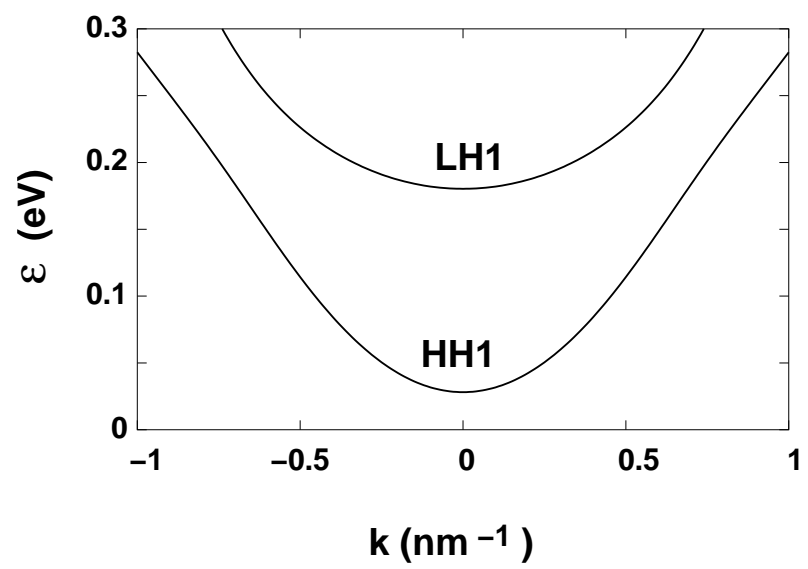

FIG. 2: Dispersion of the lowest quasi-2D subbands (HH1 and LH1) of a semiconductor quantum well when no exchange field and no spin-orbit coupling are present. The subband-bottom energies are computed using $e E_{z}$ equal to $4 \times 10^{7} \mathrm{eV} / \mathrm{m}$ and vertical masses of $m_{\mathrm{hh} z}=0.38 m_{0}$ and $m_{\mathrm{lh} z}=$ $0.09 m_{0}$

The effective masses appearing in Eq. (3) are given by

$$
\begin{aligned}
& m_{\mathrm{hh} \|}=m /\left(\gamma_{1}+\bar{\gamma}\right) \\
& m_{\mathrm{lh} \|}=m /\left(\gamma_{1}-\bar{\gamma}\right)
\end{aligned}
$$

where the two coefficients $\gamma_{1}$ and $\bar{\gamma}=\left(\gamma_{2}+\gamma_{3}\right) / 2$ are the Luttinger parameters, ${ }^{29}$ taken in the so-called axial approximation. ${ }^{30}$ In Fig. 2 we show the HH1 and the LH1 subband dispersion relations as a function of the magnitude $k$ of $2 \mathrm{D}$ wave vector.

The Rashba spin-orbit coupling arises from the structural inversion asymmetry ${ }^{12}$ due to an asymmetric confining potential. It is described by the Hamiltonian $H_{\mathrm{rs}}=\beta(\mathbf{k} \times \mathbf{E}) \cdot \mathbf{j}$. Here, $\mathbf{E}$ is the electric field due to the confining potential $V_{\text {con }}(z)$, and $\beta$ is a material parameter. ${ }^{20,21}$ In our system, $\mathbf{E}=E_{z} \hat{\mathbf{z}}$, and $H_{\mathrm{rs}}$ reads

$$
H_{\mathrm{rs}}=i \beta E_{z} k\left(\begin{array}{cccc}
0 & 0 & \frac{\sqrt{3}}{2} e^{-i \alpha} & 0 \\
0 & 0 & -e^{i \alpha} & \frac{\sqrt{3}}{2} e^{-i \alpha} \\
-\frac{\sqrt{3}}{2} e^{i \alpha} & e^{-i \alpha} & 0 & 0 \\
0 & -\frac{\sqrt{3}}{2} e^{i \alpha} & 0 & 0
\end{array}\right) \text {. }
$$

In Fig. 3] we show the HH and the LH subbands for the nonmagnetic semiconductor as a function of $k$, obtained by diagonalization of $H_{\mathrm{p}}=H_{2 \mathrm{D}}+H_{\mathrm{rs}}$. We see that the splitting at small wave vectors is linear for the light-hole subbands but cubic for the heavy-hole subbands. 
The Hamiltonian for the ferromagnetic-semiconductor part is given by $H_{\mathrm{f}}=H_{2 \mathrm{D}}+$ $H_{\mathrm{rs}}+H_{\mathrm{pd}}$. We use a phenomenological description of the ferromagnetic semiconductor, in which local moments with $S=5 / 2$ from $\mathrm{Mn}^{2+}$ ions are antiferromagnetically coupled to the itinerant holes $\frac{31.32 .33}{1}$ In a mean-field treatment, combined with a virtual-crystal approximation, $\stackrel{34.35 .36 .37}{ }$ itinerant holes experience an exchange field $\mathbf{h}=J_{\mathrm{pd}} N_{\mathrm{Mn}}\langle\mathbf{S}\rangle$, where the average Mn-ion spin $\mathbf{S}$ has direction $\hat{\mathbf{n}}=(\cos \phi \sin \theta, \sin \phi \sin \theta, \cos \theta), N_{\mathrm{Mn}}$ is the doping concentration, and $J_{\text {pd }}$ describes the coupling strength. This exchange-coupling field is accounted for in $H_{\mathrm{pd}}=\mathbf{h} \cdot \sigma$, where the spin matrices $\sigma$ in the basis given in Eq. (11) are

$$
\begin{gathered}
\sigma_{x}=\left(\begin{array}{cccc}
0 & 0 & \frac{1}{2 \sqrt{3}} & 0 \\
0 & 0 & \frac{1}{3} & \frac{1}{2 \sqrt{3}} \\
\frac{1}{2 \sqrt{3}} & \frac{1}{3} & 0 & 0 \\
0 & \frac{1}{2 \sqrt{3}} & 0 & 0
\end{array}\right), \\
\sigma_{y}=i\left(\begin{array}{cccc}
0 & 0 & -\frac{1}{2 \sqrt{3}} & 0 \\
0 & 0 & \frac{1}{3} & -\frac{1}{2 \sqrt{3}} \\
\frac{1}{2 \sqrt{3}} & -\frac{1}{3} & 0 & 0 \\
0 & \frac{1}{2 \sqrt{3}} & 0 & 0
\end{array}\right),
\end{gathered}
$$

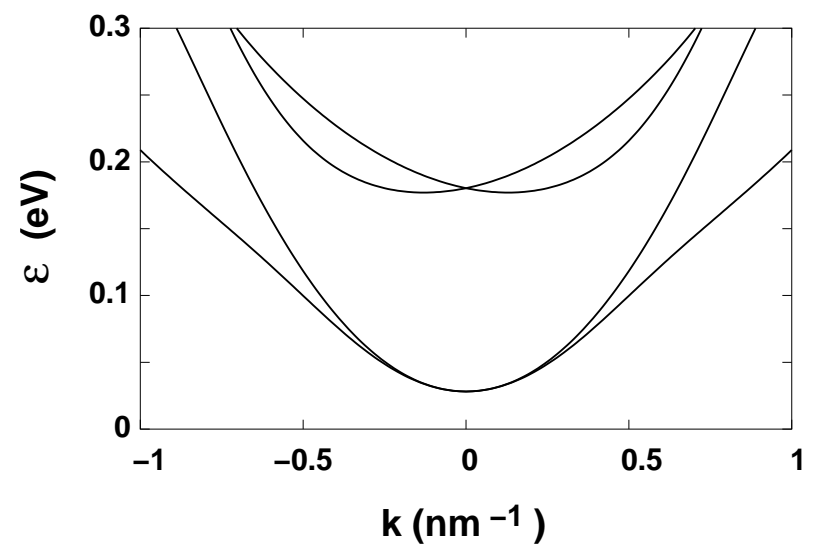

FIG. 3: The first subbands (HH1 and LH1) of a semiconductor quantum well in the presence of Rashba spin-orbit coupling, which removes the spin degeneracy. The coupling constant $\left\langle\beta E_{z}\right\rangle$ is $0.15 \mathrm{eV} \mathrm{nm}$. 


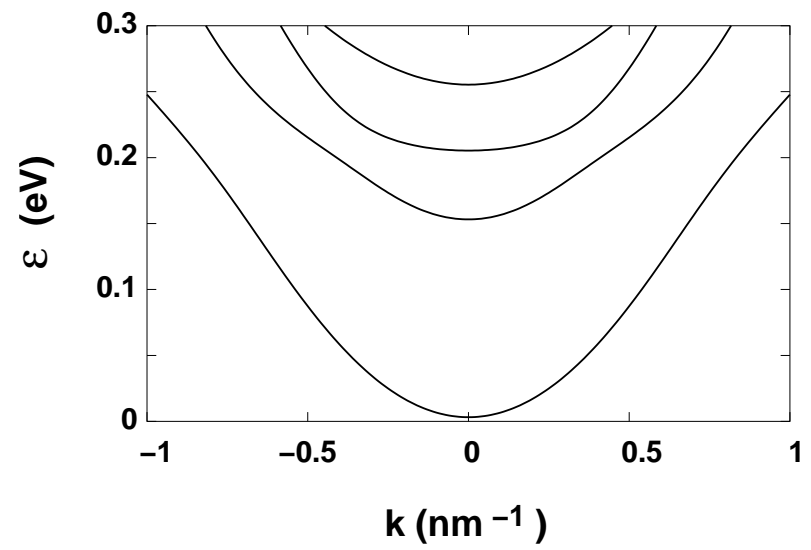

FIG. 4: The ferromagnetic-semiconductor 2D quantum-well subbands for the case of magnetization perpendicular to the 2DHG plane. The interaction constant is $J_{\mathrm{pd}}=0.06 \mathrm{eV} \mathrm{nm}^{3}$, the Manganese concentration is $N_{\mathrm{Mn}}=1 \mathrm{~nm}^{-3}$, and $\left\langle\beta E_{z}\right\rangle=0.15 \mathrm{eV} \mathrm{nm}$.

and

$$
\sigma_{z}=\left(\begin{array}{cccc}
\frac{1}{2} & 0 & 0 & 0 \\
0 & -\frac{1}{6} & 0 & 0 \\
0 & 0 & \frac{1}{6} & 0 \\
0 & 0 & 0 & -\frac{1}{2}
\end{array}\right) .
$$

In Fig. 4 we show the HH and the LH subbands in the ferromagnetic-semiconductor contacts as a function of $k$, obtained by diagonalization of $H_{\mathrm{f}}$. The splitting of the two heavy-hole subbands for small values of $k$ leads to full polarization at low densities. The magnetization direction in the Figure is perpendicular to the $2 \mathrm{DHG}$ plane, $\hat{\mathbf{n}}=(0,0,1)$.

\section{QUANTUM STATES FOR HOLES PROPAGATING THROUGH THE SPIN FET}

We calculate coherent transport through the spin FET using the scattering formalism described, e.g., in Ref. 38. It relates the current to transmission amplitudes for scattering states defined in the contacts. To obtain these, proper matching of wave functions at interfaces is required, that we describe in this section. The imposed conditions at the interface are the continuity of the wave function and conservation of the component of probability current that is perpendicular to the interface. To illustrate the subtleties associated with the second condition, let us consider an interface between a ferromagnetic and a nonmagnetic 
region at $x=x_{0}$. The continuity equation for the current is

$$
v_{x}^{\mathrm{f}} \psi^{\mathrm{f}}\left(x_{0}, y\right)=v_{x}^{\mathrm{p}} \psi^{\mathrm{p}}\left(x_{0}, y\right)
$$

with

$$
v_{x}^{\mathrm{f}, \mathrm{p}}=\frac{1}{\hbar} \frac{\partial H_{\mathrm{f}, \mathrm{p}}}{\partial k_{x}}
$$

which derives from the operator relation $\hat{v}_{x}=\frac{i}{\hbar}[\hat{H}, \hat{x}]$. Note that, due to the presence of spin-orbit coupling, the derivative of wave functions needs not to be continuous at $x=x_{0}$. Instead, Eq. (9) guarantees current conservation.

In both the ferromagnetic and nonmagnetic regions, four different channels are available, $i=1, \ldots, 4$, associated with the four-dimensional Hilbert space of the valence-band subspace under consideration. Let us consider a wave incoming from the ferromagnetic region with wave vector $\mathbf{k}_{i}^{\mathrm{I}}=k_{i}^{\mathrm{I}}(\cos \alpha, \sin \alpha)$, which is in the $i$ th subband. The wave is partially reflected at the interface to the nonmagnetic region. The wave function in the ferromagnetic source electrode is then

$$
\begin{array}{r}
\psi^{\mathrm{f}}(x, y)=\frac{\chi_{i}^{\mathrm{f}}\left(\mathbf{k}_{i}^{\mathrm{I}}\right)}{\sqrt{\left|v_{i}^{\mathrm{f}}\left(\mathbf{k}_{i}^{\mathrm{I}}\right)\right|}} e^{i k_{i}^{\mathrm{I}}(x \cos \alpha+y \sin \alpha)} \\
+\sum_{n=1}^{4} r_{i, n} \frac{\chi_{n}^{\mathrm{f}}\left(\mathbf{k}_{n}^{\mathrm{R}}\right)}{\sqrt{\left|v_{n}^{\mathrm{f}}\left(\mathbf{k}_{n}^{\mathrm{R}}\right)\right|}} e^{-i k_{n}^{\mathrm{R}}\left(x \cos \alpha_{n}^{\mathrm{R}}+y \sin \alpha_{n}^{\mathrm{R}}\right)},
\end{array}
$$

where $\chi_{n}^{\mathrm{f}}$ are the eigenfunctions of $H_{\mathrm{f}}$ in $\mathbf{k}$-space representation, the normalization factor $v_{n}^{\mathrm{f}}\left(\mathbf{k}_{n}\right)$ is the velocity expectation value computed for the state vector $\chi_{n}^{\mathrm{f}}\left(\mathbf{k}_{n}\right)$, and $r_{i, n}$ are the reflection coefficients to be determined from the matching. The wave vectors $\mathbf{k}_{n}^{\mathrm{R}}=$ $k_{n}^{\mathrm{R}}\left(\cos \alpha_{n}^{\mathrm{R}}, \sin \alpha_{n}^{\mathrm{R}}\right)$ of the reflected waves are determined by the following three conditions:

a) the modulus $k_{n}^{\mathrm{R}}$ is the solution of the implicit equation $\epsilon_{n}^{\mathrm{f}}\left(k_{n}^{\mathrm{R}}\right)=E_{\mathrm{F}}$, where $\epsilon_{n}^{\mathrm{f}}(k)$ is the dispersion relation;

b) the angles $\alpha_{n}^{\mathrm{R}}$ are derived from the continuity of the momentum parallel to the interface due to translational invariance along that spatial direction;

c) among these solutions we allow those that satisfy $\left\{\Re\left[v_{n}^{\mathrm{f}}\left(\mathbf{k}_{n}^{\mathrm{R}}\right)\right]>0 \& \Im\left[v_{n}^{\mathrm{f}}\left(\mathbf{k}_{n}^{\mathrm{R}}\right)\right]=0\right\}$ or $\left\{\Im\left[v_{n}^{\mathrm{f}}\left(\mathbf{k}_{n}^{\mathrm{R}}\right)\right]>0\right\}$. The two possibilities correspond to propagating and evanescent modes, respectively.

Similarly, the wave function in the nonmagnetic region is

$$
\psi^{\mathrm{p}}(x, y)=\sum_{n=1}^{4} t_{i, n} \frac{\chi_{n}^{\mathrm{p}}\left(\mathbf{k}_{n}^{\mathrm{T}}\right)}{\sqrt{\left|v_{n}^{\mathrm{P}}\left(\mathbf{k}_{n}^{\mathrm{T}}\right)\right|}} e^{i k_{n}^{\mathrm{T}}\left(x \cos \alpha_{n}^{\mathrm{T}}+y \sin \alpha_{n}^{\mathrm{T}}\right)},
$$


where $t_{i, n}$ are the transmission coefficients, and all the other quantities in Eq. (12) have the same definitions given for the corresponding quantities in Eq. (11). The eight coefficients $r_{i, n}$ and $t_{i, n}$ are determined by the two conditions of continuity of the wave function and current conservation, Eq. (9), given that $\psi^{\mathrm{f}, \mathrm{p}}$ has four components in the total momentum space.

We emphasize that it is important to include all modes even when some of them are evanescent since transmission and reflection at the interface are influenced by tunneling into classically forbidden channels.

\section{RESULTS OF NUMERICAL SIMULATIONS}

For our numerical simulations we take $J_{\mathrm{pd}}=0.06 \mathrm{eV} \mathrm{nm}^{3}, N_{\mathrm{Mn}}=1 \mathrm{~nm}^{-3}$ and $S=5 / 2$. The Rashba term is characterized by $\left\langle\beta E_{z}\right\rangle=0.05 \mathrm{eV} \mathrm{nm}$, and the Fermi energy is taken as $E_{\mathrm{F}}=0.08 \mathrm{eV}$, which corresponds to a hole density of $4 \times 10^{16} \mathrm{~m}^{-2}$. We assume that the leads' magnetization direction is either perpendicular $(\theta=0)$ or within the plane $(\theta=\pi / 2)$ of the quantum well.

To clarify the underlying physics, we will approach the full spin FET design step by step. First, we consider Rashba spin precession for holes transmitted through a single interface between a ferromagnetic and a nonmagnetic 2DHG for a fixed injection angle. Then, we include a second interface with the magnetization of the source and drain electrode being parallel, keeping the angle of incidence for spin-polarized holes still fixed. As a result of the Rashba effect, the total transmission will oscillate as a function of the channel length in the nonmagnetic 2DHG. Finally, we take the full 2D nature of the device into account by adding up the current contributions for all injection angles.

Let us start by considering a single interface between a ferromagnetic and a nonmagnetic 2DHG. In Fig. [5 we show the spin-up and spin-down currents as a function of the distance from the interface in the case of magnetization perpendicular to the plane $(\hat{\mathbf{n}}=(0,0,1))$ and perpendicular injection $(\alpha=0)$ of spin-up current for two different values of $E_{\mathrm{F}}$. We find that both the spin-up and spin-down current density oscillates with modulation length $L_{\text {so }}$, indicating Rashba spin precession in the nonmagnetic region. With increasing Fermi energy, the oscillation length decreases. This result is in clear contrast to the case of the spin FET

based on electrons, where the spin precession length is independent of the Fermi energy ${ }^{10}$ 

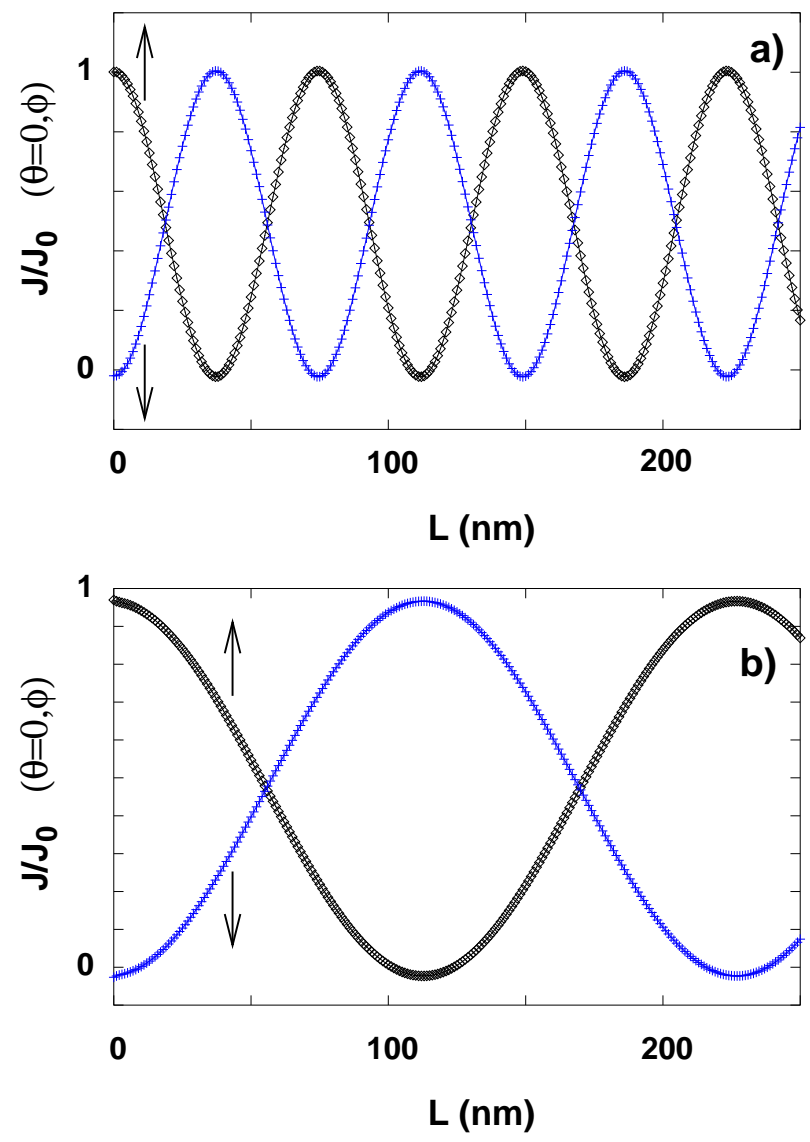

FIG. 5: Spin components of the current density in a nonmagnetic 2DHG plotted as a function of the distance $L$ from the interface with a ferromagnetic 2DHG. Results are shown for a half-metallic ferromagnetic contact having magnetization direction $\hat{\mathbf{n}}=(0,0,1)$. The Fermi energy is equal to $0.09 \mathrm{eV}$ in a) and to $0.045 \mathrm{eV}$ in panel b). It is apparent that the period of current oscillations is proportional to $1 / E_{\mathrm{F}}$. For both spin directions, the current is normalized to the incident hole flux.

Moreover, the modulation length $L_{\mathrm{so}}(\alpha)$ depends also on the injection angle $\alpha$ (not shown in Fig. 5). For a realistic sample, integration over all possible injection angles is required. We will see later that, after integration, the overall modulation length is given by that for perpendicular injection, $L_{\mathrm{so}}(0)$.

In Fig. 6] we show results for the case of magnetization direction in the ferromagnetic 2 DHG being $\hat{\mathbf{n}}=(1 / \sqrt{2}, 1 / \sqrt{2}, 0)$. For in-plane magnetization in the half-metallic contact, the amplitude of the current modulation in the nonmagnetic 2DHG depends on its azimuthal angle $\phi$. The largest oscillation amplitude occurs for $\phi=0$, i.e., when the magnetization direction is perpendicular to the interface. No oscillations exist for $\phi=\pi / 2$, because majority 


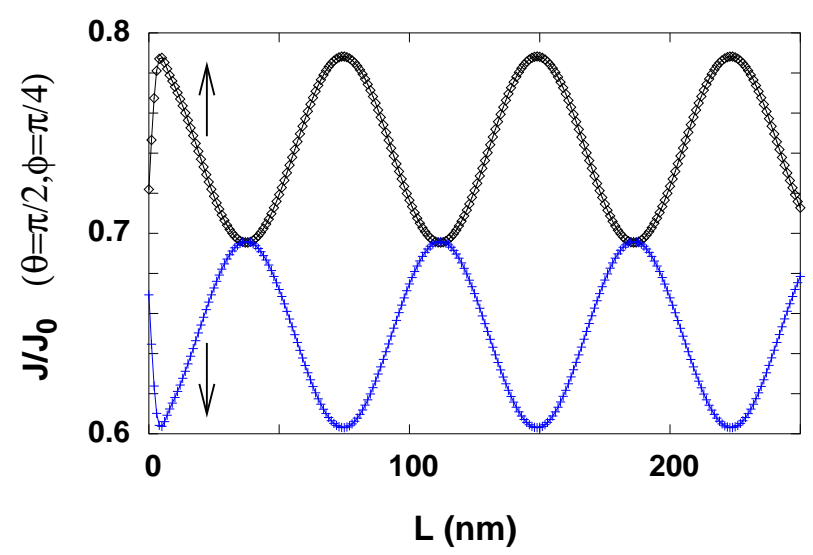

FIG. 6: Same as Fig. [5]a) but for the case where the magnetization direction in the ferromagnetic 2DHG (and, hence, the quantization axis for spin components of the current) is equal to $\hat{\mathbf{n}}=$ $(1 / \sqrt{2}, 1 / \sqrt{2}, 0)$, i.e., lies in the 2 DHG plane. Note the diminished amplitude of current oscillations which would disappear altogether for $\hat{\mathbf{n}}=(0,1,0)$.

spins injected into the nonmagnetic $2 \mathrm{DHG}$ are then eigenstates of $H_{\mathrm{rs}}$.

We now turn to the simulation of the spin FET transistor, consisting of a finite strip of nonmagnetic 2DHG with two interfaces with ferromagnetic contacts, one at $x=0$ and the other one at $x=L$. Now we have to apply the mode-matching procedure for each interface. In Fig. [7. we plot the total transmission through the entire device as a function of the width $L$ of the nonmagnetic region. We find a modulation of the transmission with modulation

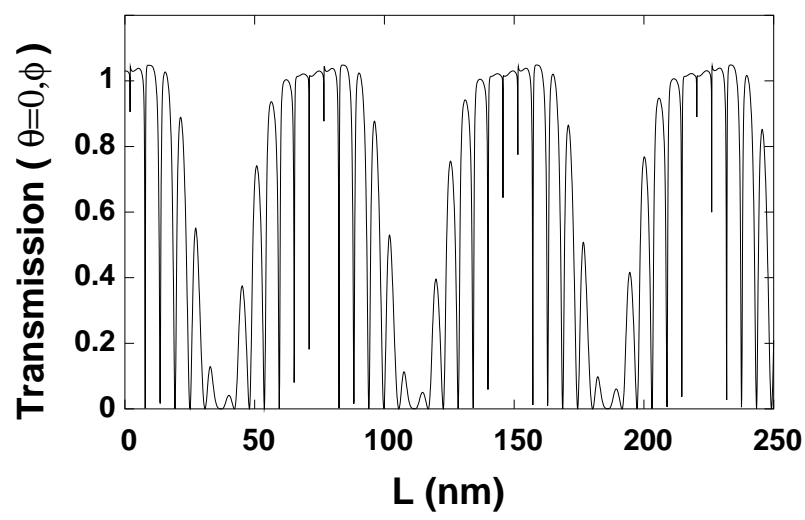

FIG. 7: The transmission probability of spin-up electrons for the case of two interfaces separated by a distance $L$. The high-frequency oscillations are due to resonances arising from multiple reflections between the two interfaces. It turns out (see below) that such features tend to be smeared out when the transmission is averaged over the injection angle. Parameters are the same as in Fig. 5. 


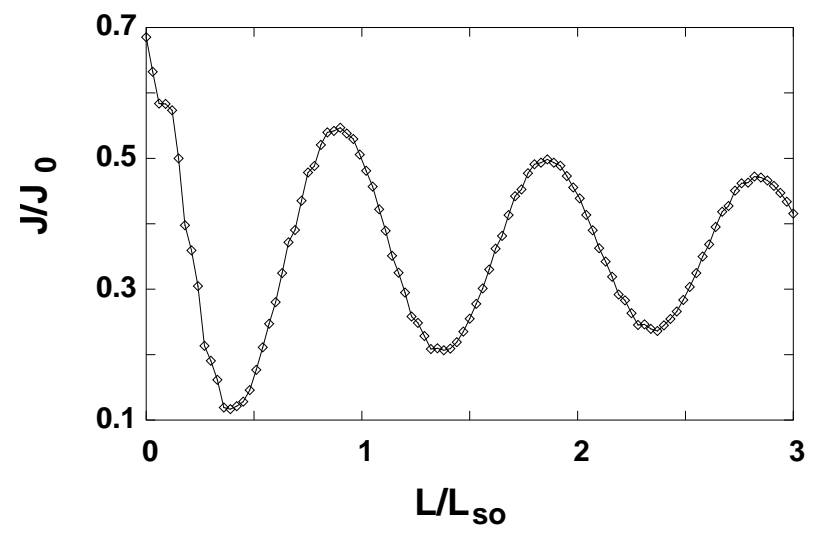

FIG. 8: The total current density as a function of the quantity $L / L_{\mathrm{so}}$ in the $2 \mathrm{D}$ system treatment, where $L$ is the channel length and $L_{\mathrm{so}}$ is the total modulation length. The magnetization in the contacts is perpendicular to the plane of the 2DHG.

length $L_{\mathrm{so}}$, which is due to Rashba spin precession. This modulation is superimposed by fast oscillations of the order of twice the Fermi wavelength $\lambda_{\mathrm{F}}$ which are due to interference effects from multiple reflection within the double-barrier structure. $\frac{39}{.9}$ As we will see below, these fast oscillations will be almost always smeared out after integration over the injection angles $\alpha$, i.e., they will not appear in real 2D devices. Only in the limit of very low hole densities, remnants of these oscillations will be visible.

Finally, we take into account the full 2D nature of the device, i.e., we add up the current contributions for all injection angles. We assume an isotropic angular distribution of injected holes since all our simulations are performed in the linear response regime. As a result, the transmitted current density $J / J_{0}$ is given by the formula

$$
J / J_{0}=\sum_{n, m} \int_{-\pi / 2}^{\pi / 2} T_{n m}(\alpha) \cos \alpha d \alpha,
$$

where $T_{n m}(\alpha)$ is the transmission probability from channel $n$ to $m$ for holes injected at an angle $\alpha$. Since the modulation length $L_{\mathrm{so}}(\alpha)$ of the transmission is $\alpha$-dependent, one might expect that the integration washes out the effects of the spin precession. It turns out, however, that oscillations are still visible, although damped. The modulation length of the resulting oscillations coincides with that for perpendicular incidence, $L_{\mathrm{so}}(0)$. In Fig. 8 we show the result of the integration operation corresponding to Eq. (13) for magnetization direction in the contact 2DHGs being $\hat{\mathbf{n}}=(0,0,1)$. 


\section{ANALYTICAL RESULTS FOR TRANSPORT}

In this section we show that most features of the numerical results presented in the previous section can be understood within a simplified analytical model. In particular, we derive analytic expressions for the precession length $L_{\text {so }}(\alpha)$ and the total current density. A similar model has been already proposed in Ref. 25, where it was used to discuss universal features of hole and electron spin precession. The approximate formulae derived in this section clearly show how the precession length depends on system parameters and, therefore, allow for a deeper understanding of the underlying physics than looking at the purely numerical results presented in the previous Section can provide.

The model is developed following some approximations that are justified a priori by physical considerations and a posteriori by the comparison between analytical and numerical results. First, we make use of the fact that for typical parameters only the lowest heavyhole subband is occupied. As an approximation we can, therefore, omit all non-conducting subbands from our model. Furthermore, we assume perfect transmission at the interfaces, i.e., we neglect reflection. All Hamiltonians are now represented as $2 \times 2$ matrices, using the basis (11) restricted to vectors $|1\rangle$ and $|4\rangle$. The off-diagonal matrix elements are obtained from perturbation theory for the degenerate case. ${ }^{40}$ The nonmagnetic $2 \mathrm{DHG}$ region is then described by

$$
H_{\mathrm{p}}=\frac{\hbar^{2} k^{2}}{2 m_{\mathrm{hh} \|}}\left(\begin{array}{ll}
1 & 0 \\
0 & 1
\end{array}\right)+i\left\langle\beta_{\mathrm{h}} E_{z}\right\rangle k^{3}\left(\begin{array}{cc}
0 & e^{-i 3 \alpha} \\
e^{i 3 \alpha} & 0
\end{array}\right),
$$

where $\beta_{\mathrm{h}}$ is proportional to the spin-orbit coupling of holes and is different from the $\beta$ defined in Eq. (15).

The corresponding eigenenergies are

$$
\epsilon_{1,2}(k)=\frac{\hbar^{2}}{2 m_{\mathrm{hh} \|}} k^{2} \pm\left\langle\beta_{\mathrm{h}} E_{z}\right\rangle k^{3}
$$

with eigenvectors

$$
\chi_{1,2}=\frac{1}{\sqrt{2}}\left(\begin{array}{c}
1 \\
\mp i e^{i 3 \alpha}
\end{array}\right) .
$$

The spin splitting of the eigenvalues (15), together with the conservation of the wave vector parallel to the interface, implies the presence of a double refraction phenomenon ${ }^{39.41}$ where a hole wave incident on the interface from the ferromagnet gives rise to two transmitted 
waves in the nonmagnetic 2 DHG having slightly different wave vectors. Their magnitudes $k_{1,2}$ are obtained from the implicit equation $\epsilon_{1,2}(k)=E_{\mathrm{F}}$. Typically, spin-orbit coupling can be treated as a perturbation, which means that we can linearize the expression of $k_{1,2}$ in the spin-orbit coupling strength, and arrive at $k_{1,2}=k_{0} \mp \Delta k / 2$. Here $k_{0}$ is the Fermi wave vector in the absence of spin-orbit coupling, and

$$
\Delta k=\left(\frac{2 m_{\mathrm{hh} \|}}{\hbar^{2}}\right)^{2}\left\langle\beta_{\mathrm{h}} E_{z}\right\rangle E_{\mathrm{F}},
$$

which explicitly depends on the Fermi energy. The corresponding angles of the transmitted waves' propagation direction with the interface normal are found, again in the limit of weak SO coupling, to be

$$
\alpha_{1,2}=\alpha_{0} \pm\left(\Delta k / 2 k_{0}\right) \tan \alpha_{0}
$$

where $\alpha_{0}$ is defined by

$$
k_{\mathrm{F}} \sin \alpha=k_{0} \sin \alpha_{0}
$$

Hence, the transmitted hole is described by the wave function

$$
c_{1} \chi_{1} e^{i k_{1}\left(x \cos \alpha_{1}+y \sin \alpha_{1}\right)}+c_{2} \chi_{2} e^{i k_{2}\left(x \cos \alpha_{2}+y \sin \alpha_{2}\right)} .
$$

By assuming a perfectly transparent interface, we can compute the coefficients $c_{1,2}$ simply by matching the wave functions in the ferromagnet and in the nonmagnetic semiconductor. At the interface at $x=L$ to the second ferromagnet, for the case of its magnetization pointing in positive $z$ direction, only the $|+\rangle$ component will be transmitted. Hence, the outgoing state in the right ferromagnet reads $e^{i k_{\mathrm{F}}(x \cos \alpha+y \sin \alpha)} \cos \left[\Delta k L /\left(2 \cos \alpha_{0}\right)\right]|+\rangle$. As a result, the transmission probability is

$$
T_{0, \phi}(\alpha)=\cos ^{2}\left[\frac{\gamma}{\cos \alpha_{0}}\right],
$$

where we have used the relation $\Delta k L / 2=\gamma$, and the dependence on $\alpha$ is through $\alpha_{0}$ via Eq. (19). In a similar way we can obtain the transmission probabilities for arbitrary magnetization direction in the ferromagnetic 2DHGs. The transmission probability for inplane magnetization reads

$$
T_{\pi / 2, \phi}(\alpha)=\cos ^{2}\left[\frac{\gamma}{\cos \alpha_{0}}\right]+\sin ^{2}\left[3 \alpha_{0}-\phi\right] \sin ^{2}\left[\frac{\gamma}{\cos \alpha_{0}}\right] .
$$

Finally, we can write the transmission for arbitrary magnetization direction as

$$
T_{\theta, \phi}(\alpha)=\cos ^{2} \theta T_{0, \phi}+\sin ^{2} \theta T_{\pi / 2, \phi} .
$$


Equations. (21]23) cease to be valid once one of the transmitted states in the nonmagnetic 2DHG becomes evanescent, i.e., is totally reflected. This condition defines critical angles $\alpha_{\mathrm{c},\{1,2\}}$, that in the limit of weak SO coupling read $\alpha_{\mathrm{c},\{1,2\}}=k_{0} / k_{\mathrm{F}} \mp \frac{1}{2} \Delta k / k_{\mathrm{F}} \approx k_{0} / k_{\mathrm{F}}=\alpha_{c}$. We note that very similar formulae for the transmission can be obtained for electrons. ${ }^{25}$

From Eqs. (21) and (22) we find the precession length

$$
L_{\mathrm{so}}(\alpha)=\frac{2 \pi}{\left\langle\beta_{\mathrm{h}} E_{z}\right\rangle}\left(\frac{\hbar^{2}}{2 m_{\mathrm{hh} \|}}\right)^{2} \frac{\cos \alpha_{0}}{E_{\mathrm{F}}}
$$

which depends on both the injection angle $\alpha$ and the Fermi energy $E_{\mathrm{F}}$. The physical reason for the latter dependence is the cubic spin splitting of the heavy-hole subband. For a 2D device under consideration in this paper, one has to integrate over all injection angles, see Eq. (13). The final result reads then

$$
\begin{aligned}
& J / J_{0}=\left\{\cos ^{2} \theta F(\gamma)+\sin ^{2} \theta\right. \\
& \left.\times\left[\sin ^{2} \phi+F(\gamma) \cos ^{2} \phi+G(\gamma) \cos (2 \phi)\right]\right\},
\end{aligned}
$$

where $J_{0}$ is the injected current density, and the functions $F(\gamma)$ and $G(\gamma)$ are defined as

$$
\begin{gathered}
F(\gamma)=\frac{1}{2} \int_{-\frac{\pi}{2}}^{\frac{\pi}{2}} \cos \alpha \cos ^{2}\left(\frac{\gamma}{\cos \alpha}\right) d \alpha \\
G(\gamma)=\frac{1}{2} \int_{-\frac{\pi}{2}}^{\frac{\pi}{2}} \cos \alpha \sin ^{2}(3 \alpha) \sin ^{2}\left(\frac{\gamma}{\cos \alpha}\right) d \alpha
\end{gathered}
$$

A good analytical approximation for $F(\gamma)$ and $G(\gamma)$ is given in Ref. 25. The result Eq. (25) describes damped oscillations of the current density as a function of the length of the nonmagnetic part, where the modulation length is given, in agreement with our numerical findings, by the precession length for perpendicular injection, $L_{\mathrm{so}}(\alpha=0)$.

\section{GATE-VOLTAGE MANIPULATION OF THE CURRENT: CAPACITANCE MODEL}

In this section we consider the response of the hole spin FET to external gate voltages and point out important differences in its behavior compared to the electron version. With standard densities of about $10^{16} \mathrm{~m}^{-2}$, only the lowest spin-split 2D HH subbands are occupied. Their $k^{3}$ spin splitting leads to an inversely linear dependence on the Fermi energy

$E_{\mathrm{F}}$ for the hole precession length $L_{\mathrm{so}}^{\mathrm{h}}$. Hence, variation of gate voltages will modify $L_{\mathrm{so}}^{\mathrm{h}}$ by changing, at the same time, the asymmetry of the hole confinement in the 2DHG and the 


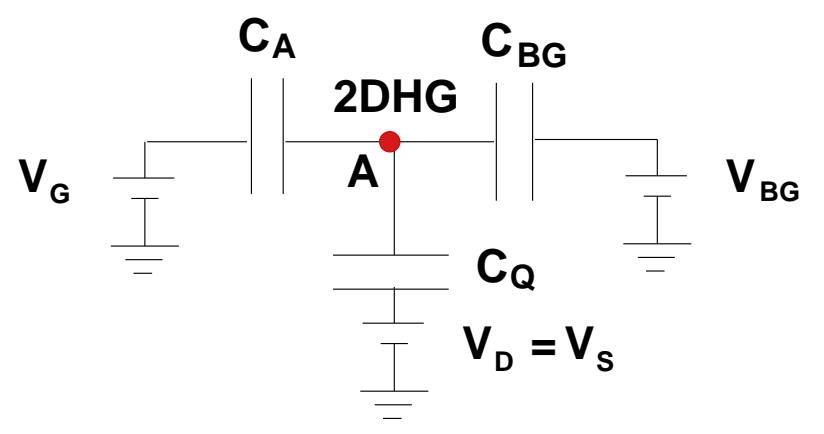

FIG. 9: Linearized capacitance model for the influence of top and back-gate voltages on the spin FET. The 2DHG is located at point $\mathrm{A}, V_{\mathrm{G}}$ is the voltage applied at the top gate, $V_{\mathrm{BG}}$ that of the back gate. The voltages at the drain $\left(V_{\mathrm{D}}\right)$ and source $\left(V_{\mathrm{S}}\right)$ contacts are assumed to be equal since we consider the linear response regime.

Fermi energy. We analyze how the device performance changes when, instead of only a top gate voltage, both top gate and back gate voltages are applied.

We model the effect of the top and back-gate voltages through a linearized capacitance model as shown in Fig. 9. The capacitance per unit area between the top gate and the point $\mathrm{A}$, where the $2 \mathrm{DHG}$ is located, is $C_{\mathrm{A}}=\epsilon_{0} \epsilon_{\mathrm{GaAs}} / d_{\mathrm{A}} \sim 2 \times 10^{-3} \mathrm{~F} / \mathrm{m}^{2}$ for an effective distance $d_{\mathrm{A}}=50 \mathrm{~nm}$ between top gate and 2DHG, while the capacitance associated to the variation of the back-gate voltage is $C_{\mathrm{BG}}=C_{\mathrm{A}} / 2$ for a distance of $100 \mathrm{~nm}$ from the $2 \mathrm{DHG}$. The capacitance between the 2DHG and the source and the drain is well approximated by

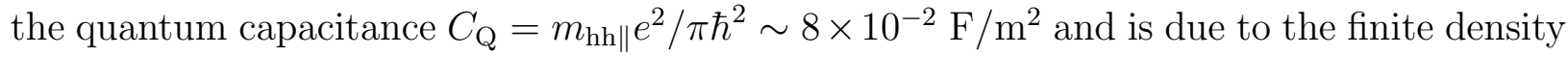
of states in the $2 \mathrm{DHG} . \stackrel{42}{=}$ The variation of the voltage at the point $\mathrm{A}$ and of the electric field $E_{z}$ read then

$$
\begin{aligned}
d V_{\mathrm{A}} & =\frac{C_{\mathrm{A}}}{C_{\mathrm{A}}+C_{\mathrm{Q}}+C_{\mathrm{BG}}} d V_{\mathrm{G}}+\frac{C_{\mathrm{BG}}}{C_{\mathrm{A}}+C_{\mathrm{Q}}+C_{\mathrm{BG}}} d V_{\mathrm{BG}} \\
d E_{z} & =-\frac{C_{\mathrm{Q}}+C_{\mathrm{BG}}}{\epsilon_{0} \epsilon_{\mathrm{GaAs}}}\left(d V_{\mathrm{A}}-d V_{\mathrm{BG}}\right) \\
& =-\frac{\left(C_{\mathrm{Q}}+C_{\mathrm{BG}}\right)\left[C_{\mathrm{A}} d V_{\mathrm{G}}-\left(C_{\mathrm{A}}+C_{\mathrm{Q}}\right) d V_{\mathrm{BG}}\right]}{\epsilon_{0} \epsilon_{\mathrm{GaAs}}\left(C_{\mathrm{A}}+C_{\mathrm{Q}}+C_{\mathrm{BG}}\right)}
\end{aligned}
$$

while the variation of the Fermi energy reads

$$
d E_{\mathrm{F}}=-e d V_{\mathrm{A}}=\frac{-e\left(C_{\mathrm{A}} d V_{\mathrm{G}}+C_{\mathrm{BG}} d V_{\mathrm{BG}}\right)}{C_{\mathrm{Q}}+C_{\mathrm{BG}}+C_{\mathrm{A}}} .
$$

It is clear that variation of only $V_{\mathrm{G}}$ (i.e., keeping $d V_{\mathrm{BG}}=0$ ) simultaneously changes $E_{z}$ and $E_{\mathrm{F}}$. In order to leave the Fermi level pinned, we have to manipulate both top and 
back-gate voltages such that $d V_{\mathrm{BG}}=-\left(C_{\mathrm{A}} / C_{\mathrm{BG}}\right) d V_{\mathrm{G}}$. Results of our simulation for the case that only the top-gate voltage is varied $\left(d V_{\mathrm{BG}}=0\right)$ is shown in Fig. 10. Here the current density obtained from Eq. (13) is plotted as function of the gate voltage $V_{\mathrm{G}}$ for the case when the magnetization direction in the contacts is perpendicular to the 2DHG. Spinprecession-induced current oscillations are clearly visible. The oscillation period actually varies with changing $V_{\mathrm{G}}$ due to the induced variation of $E_{\mathrm{F}}$, as mentioned in Sec. III Note that, for the parameters of Fig. 10, the effect of the gate voltage on the position of subband bottoms is negligible since the electric field is changed only by a few percent from its initial value $E_{z} \sim e p / \epsilon_{0} \epsilon_{\mathrm{GaAs}}$. (Here $p$ is the hole density.) For comparison, we show results for the case where both top and back-gate voltages are varied simultaneously such that the hole density in the 2DHG remains unchanged in Fig. 11]. Here the precession length changes only due to the gate-voltage-induced variation of the structural inversion asymmetry, measured here by the electric field $E_{z}$. Current oscillations have then a larger period as function of $V_{\mathrm{G}}$ than in the case where $V_{\mathrm{BG}}$ is kept constant. In both cases, however, a clear modulation of the current as a function of gate voltage $V_{\mathrm{G}}$ is obtained. These are slightly damped due to the superposition of current amplitudes for all possible angles of incidence.

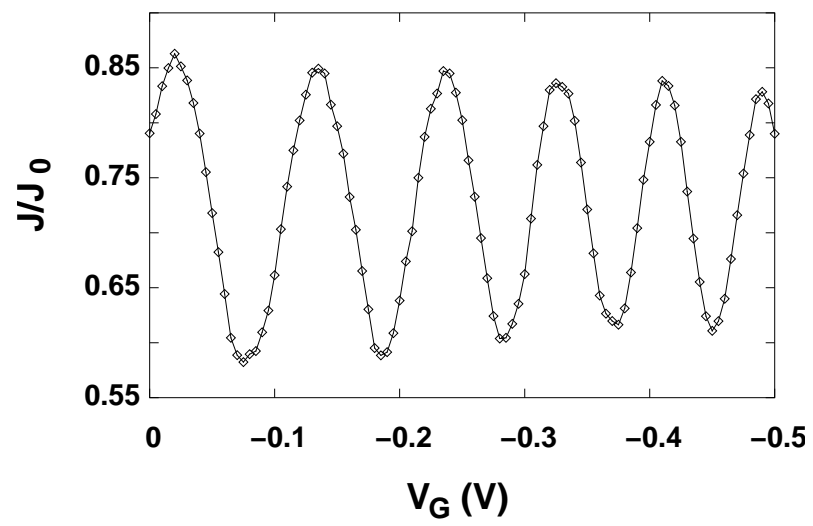

FIG. 10: The modulation of the current density through the spin FET when the top-gate voltage is varied. The magnetization direction in the ferromagnetic 2 DHGs is given by $\hat{\mathbf{n}}=(0,0,1)$. The Fermi energy for $V_{\mathrm{G}}=0$ is $E_{\mathrm{F}}=0.09 \mathrm{eV}$, the Rashba coupling term $\left\langle\beta E_{z}\right\rangle=0.05 \mathrm{eV} \mathrm{nm}$. 


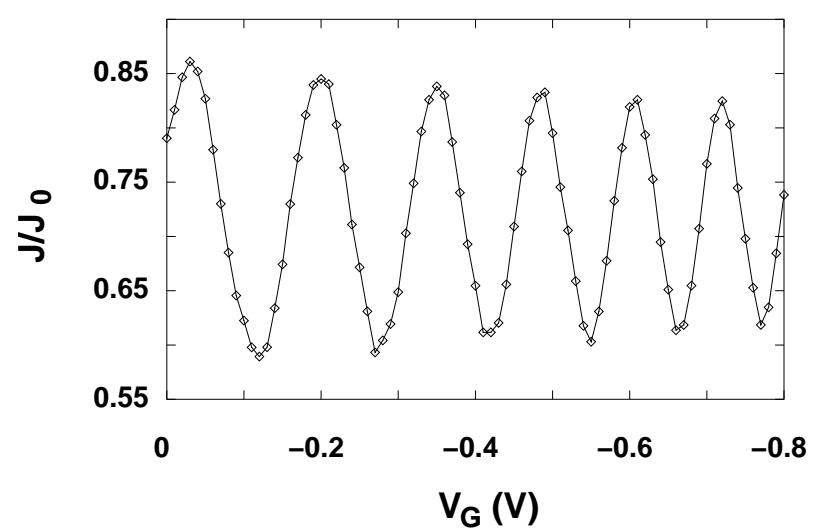

FIG. 11: The modulation of the current density in the spin FET when the top-gate voltage and the bulk-gate voltage are simultaneously varied in a way such that $E_{\mathrm{F}}$ is unchanged. The contact magnetization points along $\hat{\mathbf{n}}=(0,0,1)$.

\section{CONCLUSIONS}

We have performed careful numerical and analytical studies of transport through a p-type all-semiconductor spin FET. The design of such a device would overcome problems associated with the fabrication of hybrid devices involving metal-semiconductor contacts. Despite the more complicated nature of spin splitting in $2 \mathrm{D}$ valence-band states, clear current modulation as a function of device parameters such as the width of the nonmagnetic region are observed. Using a phenomenological model for the action of external gate voltages, we have shown the possibility of current manipulation as was envisioned in the original electron spin FET proposal by Datta and Das. ${ }^{10}$ Our numerical simulations that were performed for realistic sample parameters, as well as analytical formulae reported in this work, should serve as a useful basis for experimental realization and functional optimization of a $\mathrm{p}$-type spin FET.

\section{Acknowledgments}

This work was supported by Deutsche Forschungsgemeinschaft via the Center for Functional Nanostructures at the University of Karlsruhe, by the Emmy-Noether program, by the IST NanoTCAD project (EC contract IST-1999-10828) and by EU Research Training 
Network RTN2-2001-00440.

* Present and permanent address.

1 S. A. Wolf, D. D. Awschalom, R. A. Buhrman, J. M. Daughton, S. von Molnár, M. L. Roukes, A. Y. Chtchelkanova, and D. M. Treger, Science 294, 1488 (2001).

2 Semiconductor Spintronics and Quantum Computation, D. D. Awschalom, D. Loss, and N. Samarth (eds.), Series Nanoscience and Technology (Springer, Berlin, 2002).

3 E. A. de Andrada e Silva and G. C. La Rocca, Phys. Rev. B 59, R15583 (1999).

4 J. Nitta, F. E. Meijer, and H. Takayanagi, Appl. Phys. Lett. 75, 695 (1999).

5 A. A. Kiselev and K. W. Kim, Appl. Phys. Lett. 78, 775, (2001).

6 T. Koga, J. Nitta, H. Takayanagi, and S. Datta, Phys. Rev. Lett. 88, 126601 (2002).

7 M. Governale, D. Boese, U. Zülicke, and C. Schroll, Phys. Rev. B 65, 140403(R) (2002).

8 J. C. Egues, G. Burkard, and D. Loss, Phys. Rev. Lett. 89, 176401 (2002)

9 C. Ciuti, J. P. Mc Guire, and L. J. Sham, Appl. Phys. Lett. 81, 4781 (2002).

10 S. Datta and B. Das, Appl. Phys. Lett. 56, 665, (1990).

11 For an interesting recent modification of the original proposal, see J. Schliemann, J. C. Egues, and D. Loss, Phys. Rev. Lett. 90, 146801 (2003).

12 E. I. Rashba, Fiz. Tverd. Tela (Leningrad) 2, 1224 (1960) [Sov. Phys. Solid State 2, 1109 (1960)].

13 Y. A. Bychkov and E. I. Rashba, J. Phys. C 17, 6039 (1984).

14 G. Lommer, F. Malcher, and U. Rössler, Phys. Rev. Lett. 60, 728 (1988).

15 J. Nitta, T. Akazaki, H. Takayanagi, and T. Enoki, Phys. Rev. Lett. 78, 1335 (1997).

16 T. Schäpers, G. Engels, J. Lange, Th. Klocke, M. Hollfelder, and H. Lüth, J. Appl. Phys. 83, 4324 (1998).

17 D. Grundler, Phys. Rev. Lett. 84, 6074 (2000).

18 G. Schmidt, D. Ferrand, L. W. Molenkamp, A. T. Filip, and B. J. van Wees, Phys. Rev. B 62, R4790 (2000).

19 H. Ohno, Science 281, 951 (1998).

20 R. Winkler, Phys. Rev. B 62, 4245 (2000).

21 R. Winkler, H. Noh, E. Tutuc, and M. Shayegan, Phys. Rev. B 65, 155303 (2002).

22 L. Pfeiffer, K. W. West, H. L. Stormer, J. P. Eisenstein, K. W. Baldwin, D. Gershoni, and 
J. Spector, Appl. Phys. Lett. 56, 1697 (1990).

23 M. Grayson, Ç. Kurdak, D. C. Tsui, S. Parihar, S. Lyon, and M. Shayegan, Solid-State Electron. 40, 233 (1996).

24 J. P. Lu, J. B. Yau, S. P. Shukla, M. Shayegan, L. Wissinger, U. Rössler, and R. Winkler, Phys. Rev. Lett. 81, 1282 (1998).

25 M. G. Pala, M. Governale, J. König, and U. Zülicke, cond-mat/0212560 (2002).

26 W. W. Chow, S. W. Koch, and M. Sargent III, in Semiconductor Laser Physics (Springer-Verlag, Berlin, 1994), pp. 179-211.

27 G. Bastard, J. A. Brum, and R. Ferreira, Solid State Physics, vol. 44, Semiconductor Heterostructures and Nanostructures (Academic Press, San Diego, 1991) p. 229-416.

28 An alternative method to calculate subband dispersion relations in 2D structures is presented in J. Fernàndez-Rossier and L. J. Sham, Phys. Rev. B 64, 235323 (2001).

29 J. M. Luttinger and W. Kohn, Phys. Rev. 97, 869 (1955).

30 M. Altarelli, Heterojunctions and Semiconductor Superlattices (Springer-Verlag, Berlin, 1985).

31 T. Dietl, Semicond. Sci. Technol. 17, 377 (2002).

32 B.H. Lee, T. Jungwirth, and A. H. MacDonald, Semicond. Sci. Technol. 17, 393 (2002).

33 J. König, J. Schliemann, T. Jungwirth, and A. H. MacDonald, in Electronic Structure and Magnetism of Complex Materials, Springer Series in Material Sciences 54, (Springer, 2003), pp. 163-211;

34 T. Dietl, H. Ohno, F. Matsukura, J. Cibert, and D. Ferrand, Science 287, 1019 (2000).

35 B. Lee, T. Jungwirth, and A. H. MacDonald, Phys. Rev. B 61, 15606 (2000).

36 M. Abolfath, T. Jungwirth, J. Brum, and A. H. MacDonald, Phys. Rev. B 63, 054418 (2001).

37 T. Dietl, H. Ohno, and F. Matsukura, Phys. Rev. B 63, 195205 (2001).

38 S. Datta, Electron Transport in Mesoscopic Systems (Cambridge University Press, Cambridge, UK, 1995).

39 T. Matsuyama, C.-M. Hu, D. Grundler, G. Meier, and U. Merkt, Phys. Rev. B 65, 155322 (2002).

40 G. L. Bir and G. E. Pikus, Symmetry and Strain-Induced Effects in Semiconductors (Wiley, New York, 1974) p. 135-140.

41 V. Marigliano Ramaglia, V. Cataudella, G. De Filippis, C. A. Perroni, and F. Ventriglia, cond-mat/0203569. 
42 S. Luryi, Appl. Phys. Lett. 52, 501 (1988). 